\title{
Ergodicity of the finite and infinite dimensional $\alpha$-stable Systems
}

\author{
Lihu Xu and Bogusław Zegarliński
}

\begin{abstract}
Some finite and infinite dimensional perturbed $\alpha$-stable dynamics are constructed and studied in this paper. We prove that the finite dimensional system is strongly mixing, while in the infinite dimensional case that the functional coercive inequalities are not available, we develop and apply a technique to prove the point-wise ergodicity for systems with sufficiently small interaction in a large subspace of $\Omega=\mathbf{R}^{\mathbf{Z}^{d}}$.
\end{abstract}

Key words and phrases. Ergodic property, strongly mixing, Ornstein-Uhlenbeck $\alpha$-stable processes, spin system, finite speed of propagation.

2000 Mathematics Subject Classification. 60H10, 60H15.

\section{Introduction}

In the last several decades, $\alpha$-stable processes have been deeply studied and widely applied to physics, queueing theory, mathematical finances and others. It is well known that the Ornstein-Uhlenbeck process driven by $\alpha$-stable noise (see (1.0.1) with $b=0$ ) is ergodic ([1]), however, there seems no results on the ergodic property of perturbed OrnsteinUhlenbeck $\alpha$-stable processes defined by (1.0.1), which is the main motivation for the first part of the paper (section 2). More precisely, we will study in section 2 an $n$ dimensional perturbed Ornstein-Uhlenbeck $\alpha$-stable system

$$
\left\{\begin{array}{l}
d X_{t}=-X_{t} d t+d Z_{t}+b\left(X_{t}\right) d t \\
X_{0}=x
\end{array}\right.
$$

where $Z_{t}$ is some $\alpha$-stable process $(1<\alpha<2)$ and $b \in C_{\infty}\left(\mathbf{R}^{n}\right)$ (continuous function vanishing at infinity), and prove that the system (1.0.1) is ergodic and strongly mixing as $b$ is small (Theorem II and Corollary 2.2.1).

However, the more important goal is to study the ergodic property of some infinite dimensional system. We study in section 3 a spin system of infinite dimensional OrnsteinUhlenbeck $\alpha$-stable processes perturbed by finite range bounded interaction, i.e. for every 
$i \in \mathbf{Z}^{d}$

$$
\left\{\begin{array}{l}
d X_{i}(t)=-X_{i}(t) d t+d Z_{i}(t)+U_{i}\left(X_{\Lambda_{i}}(t)\right) d t \\
X_{i}(0)=x_{i}
\end{array}\right.
$$

where $X_{i} \in \mathbf{R}^{n}$ with $n \in \mathbf{N}, X_{\Lambda_{i}}(t)=\left(X_{j}(t)\right)_{j \in \Lambda_{i}}, U_{i}$ are some bounded cylinder functions on $C\left(\left(\mathbf{R}^{n}\right)^{\mathbf{Z}^{d}}, \mathbf{R}\right)$ (continuous space with product topology) and $\left\{Z_{i}(t)\right\}_{i \in \mathbf{Z}^{d}}$ are a sequence of independent $\alpha$-stable processes $(1<\alpha<2)$ on $\mathbf{R}^{n}$. There are two motivations to study the ergodic property of the system (1.0.2). The first one is from the work by Zegarlinski on interacting unbounded spin systems driven by an essentially cylindrical Wiener noise ([13]). The system being studied there is similar to (1.0.2) but replaces the cylindrical $\alpha$-stable noises by an essential cylindrical Brownian motion. The other motivation is related to the (quantum) lattice systems described by stochastic PDE (see section 12.3 in [6], chapter 17 in [11], and the literatures within). Chapter 17 in [11] essentially studied the lattice system as the following (see (17.1) there)

$$
d X_{k}=\left(\sum_{j \in \mathbf{Z}^{d}} a_{k j} X_{j}+f\left(X_{k}\right)\right) d t+g\left(X_{k}\right) d Z_{k}(t), \quad k \in \mathbf{Z}^{d}
$$

where $a_{k j} \in \mathbf{R}$ for $k, j \in \mathbf{Z}^{d}, f: \mathbf{R} \rightarrow \mathbf{R}$ satisfying Lipchitz condition, $g: \mathbf{R} \rightarrow \mathbf{R}$ and $Z_{k}(t)$ is some $\alpha$-stable process. (1.0.3) takes away the Brownian motion and the Poisson noise from the (17.1) in [11] because the ergodic result there is essentially about this simplified system (The ergodic results on the lattice system driven by Brownian motion have been obtained in section 12.3 of [6]).

Let us compare our approach to the ergodicity of (1.0.2) and those in [13] and [11]. (1). As mentioned before, the system in [13] is similar to (1.0.2), only replacing the $\alpha$-stable processes by Brownian motions. However, that system is reversible and thus have an a'priori given unique invariant measure $\mu$ (Gibbs measure). In the framework of $L^{2}(\mu)$, the infinitesimal generator of the system is self-adjoint, thus one easily constructs the reversible dynamics by the theory of spectral decomposition of self-adjoint operators. Moreover, people can prove the ergodic property in the uniform norm sense by the tools of functional inequalities, i.e. the logarithmic Sobolev inequality (LSI) together with spectral gap inequality implies $\left\|P_{t} f-\mu(f)\right\|_{\infty} \leq e^{-c t}\|f-\mu(f)\|_{\infty}$ where $P_{t}$ is the semigroup generated by the infinitesimal generator in [13] and $c>0$ is some constant independent of $f$ (chapter 8 of [7], [13]). Unfortunately, the system (1.0.2) is nonsymmetric and thus not reversible, so the tools of spectral decomposition for self-adjoint are not applicable. On the other hand, we are not able to prove some functional inequalities such as LSI for the system (1.0.2), thus the procedure of proving ergodic result in [13] will not wrok. Instead, we prove that our system is ergodic pointwisely in some large subset of $\left(\mathbf{R}^{n}\right)^{\mathbf{Z}^{d}}$ by gradient estimate and some delicate analysis on space and time. (2). The interactions in (1.0.3) are linear and unbounded, while those in our system $(1 . \overline{0.2})$ are bounded and can be nonlinear. The existence theorem of (1.0.3) is proven under the framework of stochastic PDE, in which some regular conditions has to be assumed (see the equation (17.3) and (iii) of Theorem 17.8 in [11]). To obtain its ergodic property, one has to assume that $A$ and $f$ are sufficiently dissipative (see (i) of Theorem 17.10). For the system (1.0.2), we will study it by the Kolmogorov equation, and construct an infinite 
dimensional semigroup from the equation in the same way as in [7] (chapter 8). As will be seen in Theorem IV, as the interactions $U$ are sufficiently small, we can obtain some ergodic result. Finally, we point out that our ergodicity result is on the level of semigroup (see Theorem IV) not in the sense that the transition probabilities converge to unique invariant measure. (It seems hard to obtain some invariant measures for our semigroup.)

The organization of the paper is as follows. In section 2, by the analytic approach, we study the Ornstein-Uhlenbeck $\alpha$-stable processes and prove that the system is ergodic if the perturbation is small (Theorem I, II and Corollary 2.2.1). In section 3, we first construct an infinite dimensional semigroup $P_{t}$ from (1.0.2) by some approximation procedure (Theorem III), then prove $P_{t}$ is pointwisely ergodic (Theorem IV). Section 2 can be read independent of section 3, while the latter will only apply lemma 2.1.1 and lemma 2.1.2 in the proof of (3.2.6). The last section is a formal but simple derivation of the formula (2.1.4).

Acknowledgements: The two authors both warmly thank Prof. Sergio Albeverio at Bonn University for his reading the paper and encouragements. L. Xu also thanks the hospitality of Hausdorff Institute for Mathematics in Bonn and his colleagues there during visiting Bonn university.

\section{Perturbed Ornstein-Uhlenbeck $\alpha$-stable Processes$$
(1<\alpha<2)
$$

For simplicity, we only study the 1-dimensional system in this section. But one can obtain the same results for the high dimensional systems by the same arguments. The following notations will be frequently used in this section.

- $\partial_{x}^{\alpha}$ : It is the generator of the $\alpha$-stable process (see (2.1.2) for more details).

- $C_{b}^{k}(\mathbf{R}):=\left\{f: \mathbf{R} \rightarrow \mathbf{R} ; f, f^{\prime}, \ldots, f^{(k)}\right.$ are all continuous and bounded $\}$

- $C_{\infty}(\mathbf{R}):=\left\{f \in C_{b}(\mathbf{R}) ; f\right.$ vanishes at infinity $\}$

- $C_{\infty}^{k}(\mathbf{R})=\left\{f \in C_{b}^{k}(\mathbf{R}) ; f, f^{\prime}, \ldots, f^{(k)} \in C_{\infty}(\mathbf{R})\right\}$.

- $C_{0}^{k}(\mathbf{R})=\left\{f \in C_{b}^{k}(\mathbf{R}) ; f\right.$ is compactly supported $\}$.

- $\|f\| \equiv \sup _{x}|f(x)|$ - the uniform norm.

\subsection{Preliminary: Ornstein-Uhlenbeck $\alpha$-stable Processes}

Ornstein-Uhlenbeck $\alpha$-stable processes is described by the following stochastic differential equation (SDE)

$$
\left\{\begin{array}{l}
d X_{t}=-X_{t} d t+d Z_{t} \\
X_{0}=x
\end{array}\right.
$$


where $Z_{t}(0<\alpha<2)$ is an $\alpha$-stable process with infinitesimal generator $\partial_{x}^{\alpha}$ (fractional Laplacian, [1]) defined by

$$
\partial_{x}^{\alpha} f(x)=\frac{1}{C_{\alpha}} \int_{\mathbf{R} \backslash\{0\}} \frac{f(y+x)-f(x)}{|y|^{\alpha+1}} d y, \quad C_{\alpha}=-\int_{\mathbf{R} \backslash\{0\}}(\cos y-1) \frac{d y}{|y|^{1+\alpha}} .
$$

Moreover, if $f$ has Fourier transform defined by

$$
\hat{f}(\lambda)=\frac{1}{\sqrt{2 \pi}} \int_{\mathbf{R}} f(x) e^{-i \lambda x} d x, \quad f(x)=\frac{1}{\sqrt{2 \pi}} \int_{\mathbf{R}} \hat{f}(\lambda) e^{i \lambda x} d \lambda,
$$

then

$$
\partial_{x}^{\alpha} f(x)=\frac{1}{\sqrt{2 \pi}} \int_{\mathbf{R}}|\lambda|^{\alpha} \hat{f}(\lambda) e^{i \lambda x} d \lambda .
$$

It is well known that the corresponding Kolmogorov backward equation of (2.1.1) is

$$
\left\{\begin{array}{l}
\partial_{t} u=\partial_{x}^{\alpha} u-x \partial_{x} u \\
u(0)=f
\end{array}\right.
$$

where $f$ is the initial data. If the solution of (2.1.3) is given, we can recover from it the information of stochastic process of (2.1.1). The following lemma gives the formula for the solution of (2.1.3), which will also be formally derived in a simple way in the appendix.

\section{Lemma 2.1.1. (Formula for the solution of (2.1.3))}

Suppose $f \in C_{\infty}(\mathbf{R})$, then

$$
u(t)=\int_{\mathbf{R}} p\left(\frac{1-e^{-\alpha t}}{\alpha} ; e^{-t} x, y\right) f(y) d y
$$

is a solution of (2.1.3) where $p(t ; x, y)$ is the transition probability density of the stochastic processes $\left\{Z_{t}\right\}_{t \geq 0} . \forall f \in C_{\infty}(\mathbf{R})$, set

$$
S_{t} f(x)=\int_{\mathbf{R}} p\left(\frac{1-e^{-\alpha t}}{\alpha} ; e^{-t} x, y\right) f(y) d y,
$$

$S_{t}$ is a Markov semigroup on $C_{\infty}(\mathbf{R})$.

Proof. It is well known that

$$
p(t ; x, y)=\frac{1}{\sqrt{2 \pi}} \int_{\mathbf{R}} \frac{1}{\sqrt{2 \pi}} e^{-t|\lambda|^{\alpha}+i(x-y) \lambda} d \lambda
$$

and

$$
\partial_{t} p(t ; x, y)=\partial_{x}^{\alpha} p(t ; x, y)=\partial_{y}^{\alpha} p(t ; x, y) .
$$

Setting $s(t)=\frac{1-e^{-\alpha t}}{\alpha}, z(t, x)=e^{-t} x$, and noticing the following facts

$$
\begin{aligned}
& z \partial_{z} p(s ; z, y)=x \partial_{x} p\left(s ; e^{-t} x, y\right), e^{-\alpha t} \partial_{z}^{\alpha} p(s ; z, y)=\partial_{x}^{\alpha} p\left(s ; e^{-t} x, y\right), \\
& \partial_{t} p(s ; z, y)=\partial_{s} p(s ; z, y) e^{-\alpha t}-z \partial_{z} p(s ; z, y),
\end{aligned}
$$


one can easily have

$$
\partial_{t} \int_{\mathbf{R}} p(s ; z, y) f(y) d y=\partial_{x}^{\alpha} \int_{\mathbf{R}} p\left(s ; e^{-t} x, y\right) f(y) d y-x \partial_{x} \int_{\mathbf{R}} p\left(s ; e^{-t} x, y\right) f(y) d y .
$$

To prove the semigroup property, it is sufficient to prove

$$
\int_{\mathbf{R}} p\left(\frac{1-e^{-\alpha s}}{\alpha} ; e^{-s} x, z\right) p\left(\frac{1-e^{-\alpha t}}{\alpha} ; e^{-t} z, y\right) d z=p\left(\frac{1-e^{-\alpha(s+t)}}{\alpha} ; e^{-(s+t)} x, y\right)
$$

Indeed, from (2.1.5) and applying Parseval's Theorem on Fourier transform, we have

$$
\begin{aligned}
p\left(\frac{1-e^{-\alpha t}}{\alpha} ; e^{-t} x, y\right) & =\frac{1}{\sqrt{2 \pi}} \int_{\mathbf{R}} \frac{1}{\sqrt{2 \pi}} \exp \left\{-\frac{1-e^{-\alpha t}}{\alpha}|\lambda|^{\alpha}+i\left(e^{-t} x-y\right) \lambda\right\} d \lambda \\
& =\frac{1}{\sqrt{2 \pi}} \int_{\mathbf{R}} \frac{1}{\sqrt{2 \pi}} \exp \left\{-\frac{1-e^{-\alpha t}}{\alpha} e^{\alpha t}|\lambda|^{\alpha}-i e^{t} y \lambda\right\} e^{t} e^{i \lambda x} d \lambda,
\end{aligned}
$$

and

$$
\begin{aligned}
& \int_{\mathbf{R}} p\left(\frac{1-e^{-\alpha s}}{\alpha} ; e^{-s} x, z\right) p\left(\frac{1-e^{-\alpha t}}{\alpha} ; e^{-t} z, y\right) d z \\
= & \int_{\mathbf{R}} \frac{1}{\sqrt{2 \pi}} \exp \left\{-\frac{1-e^{-\alpha s}}{\alpha}|\lambda|^{\alpha}-i e^{-s} x \lambda\right\} \frac{1}{\sqrt{2 \pi}} \exp \left\{-\frac{1-e^{-\alpha t}}{\alpha}\left|e^{t} \lambda\right|^{\alpha}-i y e^{t} \lambda\right\} e^{t} d \lambda \\
= & \frac{1}{\sqrt{2 \pi}} \int_{\mathbf{R}} \frac{1}{\sqrt{2 \pi}} \exp \left\{-\frac{1-e^{-\alpha(s+t)}}{\alpha}|\lambda|^{\alpha}+i\left(e^{-s-t} x-y\right) \lambda\right\} d \lambda \\
= & p\left(\frac{1-e^{-\alpha(s+t)}}{\alpha} ; e^{-(s+t)} x, y\right)
\end{aligned}
$$

Moreover, by the heat kernel estimate of $p(t ; x, y)$ in [5]

$$
p(t ; x, y) \leq \frac{K t}{|y-x|^{1+\alpha}}
$$

with some constant $K \in(0, \infty)$, we have

$$
\begin{aligned}
\left|S_{t} f(x)\right| & \leq \int_{|y| \leq B} p\left(\frac{1-e^{-\alpha t}}{\alpha} ; e^{-t} x, y\right)|f(y)| d y+\int_{|y|>B} p\left(\frac{1-e^{-\alpha t}}{\alpha} ; e^{-t} x, y\right)|f(y)| d y \\
& \leq \sup _{|y|>B}|f(y)|+\int_{|y| \leq B} \frac{K}{\left|y-e^{-t} x\right|^{1+\alpha}} d y|| f||
\end{aligned}
$$

where $B>0$ is sufficiently large. From (2.1.13) and the fact $f \in C_{\infty}(\mathbf{R})$, it is easy to claim that

$$
\lim _{x \rightarrow \infty} S_{t} f(x)=0
$$

thus $S_{t} f \in C_{\infty}(\mathbf{R})$. It is easy to check $S_{t} 1=1$ and that $S_{t} f \geq 0$ if $f \geq 0$. Thus $\left(S_{t}\right)_{t \geq 0}$ is a Markov semigroup on $C_{\infty}(\mathbf{R})$. 
Lemma 2.1.2. (Gradient estimates for $\left.S_{t} f\right) \forall f \in C_{\infty}^{1}(\mathbf{R})$,

$$
\begin{gathered}
\left\|\partial_{x} S_{t} f\right\| \leq e^{-t}\left\|\partial_{x} f\right\| \\
\left\|\partial_{x} S_{t} f\right\| \leq \frac{\hat{C} e^{-t}}{t^{\frac{1}{\alpha}} \wedge 1}\|f\|
\end{gathered}
$$

where $\hat{C}$ is some constant independent of $f$.

Proof. Recall

$$
S_{t} f(x)=\frac{1}{2 \pi} \int_{-\infty}^{\infty} \int_{-\infty}^{\infty} \exp \left\{-\frac{1-e^{-\alpha t}}{\alpha}|\lambda|^{\alpha}+i e^{-t} \lambda x-i \lambda y\right\} d \lambda f(y) d y
$$

we have

$$
\begin{aligned}
\left|\partial_{x} S_{t} f(x)\right| & =\left|\frac{1}{2 \pi} \int_{-\infty}^{\infty} \int_{-\infty}^{\infty} \partial_{x} \exp \left\{-\frac{1-e^{-\alpha t}}{\alpha}|\lambda|^{\alpha}+i e^{-t} \lambda x-i \lambda y\right\} d \lambda f(y) d y\right| \\
& =\left|e^{-t} \frac{1}{2 \pi} \int_{-\infty}^{\infty} \int_{-\infty}^{\infty} \partial_{y} \exp \left\{-\frac{1-e^{-\alpha t}}{\alpha}|\lambda|^{\alpha}+i e^{-t} \lambda x-i \lambda y\right\} d \lambda f(y) d y\right| \\
& =\left|e^{-t} \frac{1}{2 \pi} \int_{-\infty}^{\infty} \int_{-\infty}^{\infty} \exp \left\{-\frac{1-e^{-\alpha t}}{\alpha}|\lambda|^{\alpha}+i e^{-t} \lambda x-i \lambda y\right\} d \lambda \partial_{y} f(y) d y\right| \\
& \leq e^{-t}|| \partial_{x} f||,
\end{aligned}
$$

and

$$
\begin{aligned}
\left|\partial_{x} S_{t} f(x)\right| & =\left|\int_{-\infty}^{\infty} \int_{-\infty}^{\infty} \partial_{x} \exp \left\{-\frac{1-e^{-\alpha t}}{\alpha}|\lambda|^{\alpha}+i e^{-t} \lambda x-i \lambda y\right\} d \lambda f(y) d y\right| \\
& \leq e^{-t} \int_{-\infty}^{\infty}\left|\int_{-\infty}^{\infty} \lambda \exp \left\{-\frac{1-e^{-\alpha t}}{\alpha}|\lambda|^{\alpha}+i e^{-t} \lambda x-i \lambda y\right\} d \lambda\right| d y \cdot\|f\| \\
& =\frac{e^{-t}}{\left(\frac{1-e^{-\alpha t}}{\alpha}\right)^{\frac{1}{\alpha}}} \int_{-\infty}^{\infty}\left|\int_{-\infty}^{\infty} \lambda^{\prime} \exp \left\{-\left|\lambda^{\prime}\right|^{\alpha}-i \lambda^{\prime} y^{\prime}\right\} d \lambda^{\prime}\right| d y^{\prime} \cdot\|f\| \\
& \leq \frac{e^{-t} C}{t^{\frac{1}{\alpha}} \wedge 1}\|f\|
\end{aligned}
$$

where $\lambda^{\prime}=\left(\frac{1-e^{-\alpha t}}{\alpha}\right)^{\frac{1}{\alpha}}, y^{\prime}=\left(\frac{1-e^{-\alpha t}}{\alpha}\right)^{-\frac{1}{\alpha}}\left(y-e^{-t} x\right)$ and $\int_{-\infty}^{\infty}\left|\int_{-\infty}^{\infty} \lambda^{\prime} \exp \left\{-\left|\lambda^{\prime}\right|^{\alpha}-i \lambda^{\prime} y^{\prime}\right\} d \lambda^{\prime}\right| d y^{\prime}<$ $\infty$ is easy to check. 


\subsection{Description and Main results of finite dimensional systems}

In this section, we study the perturbed Ornstein-Uhlenbeck $\alpha$-stable system described by the SDE (1.0.1) whose Kolmogorov backward equation is well known as follows (cf. chapter $5,[3])$

$$
\left\{\begin{array}{l}
\partial_{t} u=\partial_{x}^{\alpha} u-x \partial_{x} u+b(x) \partial_{x} u \\
u(0)=f
\end{array}\right.
$$

where $b \in C_{\infty}(\mathbf{R})$. Formally the mild solution of the above equation is

$$
u(t, x)=S_{t} f(x)+\int_{0}^{t} S_{t-s}\left[b \partial_{x} u(s)\right](x) d s .
$$

Define

$$
\mathcal{L}_{1}=\partial_{x}^{\alpha}-x \partial_{x}+b(x) \partial_{x}
$$

the main results of section 2 are:

Theorem I. Suppose $1<\alpha<2$. The operator $\left(\mathcal{L}_{1}, C_{0}^{\infty}(\mathbf{R})\right)$ is closable in $C_{\infty}(\mathbf{R})$, and the closed extension generates a Markov semigroup $\left\{P_{t}\right\}_{t \geq 0}$ on $C_{\infty}(\mathbf{R})$. Moreover, $\forall f \in C_{\infty}^{2}(\mathbf{R}), P_{t} f$ is the unique mild solution of (2.2.1) and also its classical solution.

Theorem II. Suppose that $\left\{P_{t}\right\}_{t \geq 0}$ is the semigroup in Theorem I and that $C_{1}=\hat{C} \cdot\|b\| \cdot$ $\Gamma\left(1-\frac{1}{\alpha}\right)$ with $\hat{C}$ being the constant in lemma 2.1.2 and $\Gamma$ being the Gamma-function. If $\|b\|$ is sufficiently small so that

$$
C_{1}\left(1+\left[\frac{\alpha}{\alpha-1}\right]\right) e^{C_{1}}<e-1
$$

then, $\forall f \in C_{\infty}(\mathbf{R})$, there exists a constant $c$ (independent of $x$ ) such that

$$
\lim _{t \rightarrow \infty} P_{t} f(x)=c
$$

Corollary 2.2.1. Theorem II implies that there exists some probability measure $\mu$ such that

$$
\lim _{t \rightarrow \infty} p(t ; x, d y) \rightarrow \mu
$$

where $p(t ; x, d y)$ is the transition probability of the system (1.0.1). Moreover, the system (1.0.2) is strongly mixing.

Remark 2.2.2. It is easy to see that our result implies $\lim _{T \rightarrow \infty} \frac{1}{T} \int_{0}^{T} p(t ; x, d y) d t \rightarrow \mu$ weakly, which is the sense of the usual ergodic property.

\subsection{Lemmas}

Lemma 2.3.1. If $f \in C_{\infty}^{1}(\mathbf{R})$, then (2.2.1) has a unique mild solution $u$ with $u(t) \in$ $C_{\infty}(\mathbf{R})$ for every $0 \leq t<\infty$. Moreover, $u$ is also a classical solution. 
Proof. We apply Banach fixed point theorem. For some $T>0$ (to be selected later), define

$$
\mathcal{C}_{T}^{1}=\left\{u \in C\left([0, T] ; C^{1}(\mathbf{R})\right): u(0)=f, \sup _{0 \leq t \leq T}\|u(t)\|_{C^{1}}<\infty\right\}
$$

and

$$
\|u\|_{T}=\sup _{0 \leq t \leq T}\|u(t)\|_{C^{1}},
$$

$\left(\mathcal{C}_{T}^{1},\|\cdot\|_{T}\right)$ is obviously a Banach space. We consider the map $\mathcal{F}$ :

$$
\mathcal{F}[u](t)=S_{t} f+\int_{0}^{t} S_{t-s}\left[b \partial_{x} u(s)\right] d s \quad(0 \leq t \leq T)
$$

Point $1 \mathcal{F}$ is a map from $\mathcal{C}_{T}^{1}$ to $\mathcal{C}_{T}^{1}: \forall u \in \mathcal{C}_{T}^{1}$, one has

$$
\|\mathcal{F}[u](t)\| \leq\|f\|+\int_{0}^{t} \| S_{t-s}\left(b \partial_{x} u(s)\|d s \leq\| f\left\|_{C^{1}}+\right\| b\left\|\int_{0}^{t}\right\| u(s) \|_{C^{1}} d s\right.
$$

and has by applying (2.1.15) and (2.1.16)

$$
\begin{aligned}
\left\|\partial_{x} \mathcal{F}[u](t)\right\| & \leq e^{-t}\left\|\partial_{x} f\right\|+\int_{0}^{t} \frac{e^{-(t-s)} \hat{C}}{(t-s)^{\frac{1}{\alpha}} \wedge 1}\|b\| \cdot\left\|\partial_{x} u(s)\right\| d s \\
& \leq e^{-t}\|f\|_{C^{1}}+\|b\| \int_{0}^{t} \frac{e^{-(t-s)} \hat{C}}{(t-s)^{\frac{1}{\alpha}} \wedge 1}\|u(s)\|_{C^{1}} d s
\end{aligned}
$$

Hence, for $0 \leq t \leq T$,

$$
\|\mathcal{F}[u](t)\|_{C^{1}} \leq\left(2+T \cdot\|b\|+\sup _{0 \leq t \leq T} \int_{0}^{t} \frac{e^{-(t-s)} \hat{C}}{(t-s)^{\frac{1}{\alpha}} \wedge 1} d s\|b\|\right) \sup _{0 \leq t \leq T}\|u(t)\|_{C^{1}}
$$

Point $2 \mathcal{F}$ is a contraction map: $\forall w, v \in \mathcal{C}_{T}^{1}$, by the same arguments as in (2.3.2) and in (2.3.3) respectively, we have

$$
\|\mathcal{F}[w](t)-\mathcal{F}[v](t)\| \leq\|b\| \int_{0}^{t}\|w(s)-v(s)\|_{C^{1}} d s \leq\|b\| T \sup _{0 \leq t \leq T}\|w(t)-v(t)\|_{C^{1}}
$$

and

$$
\left\|\partial_{x} \mathcal{F}[w](t)-\partial_{x} \mathcal{F}[v](t)\right\| \leq\|b\| \sup _{0 \leq t \leq T} \int_{0}^{t} \frac{e^{-(t-s)} \hat{C}}{(t-s)^{\frac{1}{\alpha}} \wedge 1} d s \sup _{0 \leq t \leq T}\|w(t)-v(t)\|_{C^{1}} .
$$

Thus

$$
\left\|\partial_{x} \mathcal{F}[w](t)-\partial_{x} \mathcal{F}[v](t)\right\|_{C^{1}} \leq\|b\|\left(T+\sup _{0 \leq t \leq T} \int_{0}^{t} \frac{e^{-(t-s)} \hat{C}}{(t-s)^{\frac{1}{\alpha}} \wedge 1} d s\right) \sup _{0 \leq t \leq T}\|w(t)-v(t)\|_{C^{1}}
$$


When $T$ is small enough,

$$
\|b\|\left(T+\sup _{0 \leq t \leq T} \int_{0}^{t} \frac{e^{-(t-s)} \hat{C}}{(t-s)^{\frac{1}{\alpha}} \wedge 1} d s\right)<1 .
$$

Combining Point 1 and Point 2, and applying Banach fixed point theorem, we have a unique $u \in \mathcal{C}_{T}^{1}$ such that

$$
u(t)=S_{t} f+\int_{0}^{t} S_{t-s}\left[b \partial_{x} u(s)\right] d s \quad(0 \leq t \leq T) .
$$

By exactly the same procedure as the above on the dynamics at $[T, 2 T],[2 T, 3 T], \ldots$, we finally obtain a unique global mild solution on $C\left([0, \infty) ; C^{1}(\mathbf{R})\right)$.

From the facts of $f, b \in C_{\infty}(\mathbf{R})$, and (2.3.9) (which will be proven in the next lemma), we have $u(t) \in C_{\infty}(\mathbf{R})$, by applying the same argument as proving (2.1.14) on the mild solution $u(t)=S_{t} f+\int_{0}^{t} S_{t-s}\left[b \partial_{x} u(s)\right] d s$. It is easy to check this mild solution is also a classical solution.

Lemma 2.3.2. (Gradient estimate for $u(t)$ ) Suppose that $u$ and $f$ are the same as in Lemma 2.3.1. There exists some constant $A>0$, independent of $f$ and $u$, such that

$$
\left\|\partial_{x} u(t)\right\| \leq e^{-t} A^{t+1}\left\|\partial_{x} f\right\| .
$$

Moreover, if $\|b\| \leq \frac{1}{\hat{C} \Gamma\left(1-\frac{1}{\alpha}\right)}$, then

$$
\left\|\partial_{x} u(t)\right\| \leq e^{C_{2}} e^{-\left(1-C_{2}\right) t}|| \partial_{x} f \|
$$

where $\hat{C}$ is the constant in Lemma 2.1.2, $C_{2}=\log \left\{1+C_{1}\left(1+\left[\frac{\alpha}{\alpha-1}\right]\right) e^{C_{1}}\right\}$ and $C_{1}=$ $\hat{C} \cdot\|b\| \cdot \Gamma\left(1-\frac{1}{\alpha}\right)$.

Remark 2.3.3. When $\|b\|$ is small, $C_{1}$ and $C_{2}$ are also small, and thus $\left\|\partial_{x} u(t)\right\|$ decays exponentially.

Proof. Noticing (2.2.2), (2.1.15) and (2.1.16), one has

$$
\left\|\partial_{x} u(t)\right\| \leq e^{-t}|| \partial_{x} f\left\|+\int_{0}^{t} \frac{e^{-(t-s)} \hat{C} \cdot\|b\|}{(t-s)^{\frac{1}{\alpha}} \wedge 1}\right\| \partial_{x} u(s) \| d s
$$

Set $v(t)=e^{t} \partial_{x} u(t),(2.3 .11)$ is equivalent to

$$
\|v(t)\| \leq\left\|\partial_{x} f\right\|+\int_{0}^{t} \frac{\hat{C} \cdot\|b\|}{(t-s)^{\frac{1}{\alpha}}}\|v(s)\| d s .
$$


Iterating (2.3.12), we have

$$
\begin{aligned}
\|v(t)\| & \leq\left\|\partial_{x} f\right\|+\int_{0}^{t} \frac{\hat{C} \cdot\|b\|}{(t-s)^{\frac{1}{\alpha}}}\|v(s)\| d s \leq \sum_{k \geq 0}(\hat{C} \cdot\|b\|)^{k} \int_{0}^{t} \frac{d t_{1}}{\left(t-t_{1}\right)^{\frac{1}{\alpha}}} \cdots \int_{0}^{t_{k-1}} \frac{d t_{k}}{\left(t_{k-1}-t_{k}\right)^{\frac{1}{\alpha}}}\left\|\partial_{x} f\right\| \\
& =\sum_{k \geq 0} \frac{C_{1}^{k}}{\Gamma\left(1+k\left(1-\frac{1}{\alpha}\right)\right)} t^{k\left(1-\frac{1}{\alpha}\right)}\left\|\partial_{x} f\right\| \quad\left(\text { where } C_{1}=\hat{C} \cdot\|b\| \cdot \Gamma\left(1-\frac{1}{\alpha}\right)\right)
\end{aligned}
$$

since

$$
\begin{aligned}
& \int_{0}^{t} \frac{d t_{1}}{\left(t-t_{1}\right)^{\frac{1}{\alpha}}} \cdots \int_{0}^{t_{k-1}} \frac{d t_{k}}{\left(t_{k-1}-t_{k}\right)^{\frac{1}{\alpha}}}=\int_{0}^{t} \frac{d t_{1}}{\left(t-t_{1}\right)^{\frac{1}{\alpha}}} \cdots \int_{0}^{t_{k-2}} \frac{t_{k-1}^{1-\frac{1}{\alpha}} d t_{k-1}}{\left(t_{k-2}-t_{k-1}\right)^{\frac{1}{\alpha}}} \int_{0}^{1} \frac{d s_{k}}{\left(1-s_{k}\right)^{\frac{1}{\alpha}}} \\
= & \int_{0}^{t} \frac{d t_{1}}{\left(t-t_{1}\right)^{\frac{1}{\alpha}}} \cdots \int_{0}^{t_{k-3}} \frac{t_{k-2}^{2\left(1-\frac{1}{\alpha}\right)} d t_{k-2}}{\left(t_{k-3}-t_{k-2}\right)^{\frac{1}{\alpha}}} \int_{0}^{1} \frac{s_{k-1}^{1-\frac{1}{\alpha}} d s_{k-1}}{\left(1-s_{k-1}\right)^{\frac{1}{\alpha}}} B\left(1,1-\frac{1}{\alpha}\right) \\
= & \int_{0}^{t} \frac{d t_{1}}{\left(t-t_{1}\right)^{\frac{1}{\alpha}}} \cdots \int_{0}^{t_{k-4}} \frac{t_{k-3}^{3\left(1-\frac{1}{\alpha}\right)} d t_{k-3}}{\left(t_{k-4}-t_{k-3}\right)^{\frac{1}{\alpha}}} \int_{0}^{1} \frac{s_{k-2}^{2\left(1-\frac{1}{\alpha}\right)} d s_{k-2}}{\left(1-s_{k-2}\right)^{\frac{1}{\alpha}}} B\left(1+1-\frac{1}{\alpha}, 1-\frac{1}{\alpha}\right) B\left(1,1-\frac{1}{\alpha}\right) \\
= & t^{k\left(1-\frac{1}{\alpha}\right)} \prod_{i=0}^{k-1} B\left(1+i\left(1-\frac{1}{\alpha}\right), 1-\frac{1}{\alpha}\right) \\
= & \left.t^{k\left(1-\frac{1}{\alpha}\right)} \frac{\Gamma^{k}\left(1-\frac{1}{\alpha}\right)}{\Gamma\left(1+k\left(1-\frac{1}{\alpha}\right)\right)} \quad\left(\text { noticing } B(\alpha, \beta)=\frac{\Gamma(\alpha) \Gamma(\beta)}{\Gamma(\alpha+\beta)}\right)\right)
\end{aligned}
$$

Noticing the facts of $1-\frac{1}{\alpha}>0, \Gamma(z+1)=z \Gamma(z)$, and $\Gamma(\alpha) \geq \Gamma(1)=1$, we have

$$
\begin{aligned}
\Gamma\left(1+k\left(1-\frac{1}{\alpha}\right)\right) & =\left(k-\frac{k}{\alpha}\right)\left(k-\frac{k}{\alpha}-1\right) \ldots\left(k-\frac{k}{\alpha}-\left[k-\frac{k}{\alpha}\right]+1\right) \Gamma\left(k-\frac{k}{\alpha}-\left[k-\frac{k}{\alpha}\right]+1\right) \\
& \geq\left[k-\frac{k}{\alpha}\right] !
\end{aligned}
$$

and have by combining (2.3.13) and (2.3.15)

$$
\|v(t)\| \leq \sum_{k \geq 0} \frac{C_{1}^{k}}{\left[k-\frac{k}{\alpha}\right] !} t^{k\left(1-\frac{1}{\alpha}\right)}\left\|\partial_{x} f\right\| \quad(0 \leq t \leq 1) .
$$

Set

$$
A_{1}=\left(C_{1}+1\right)^{\frac{\alpha}{\alpha-1}}, A=1+\left(1+\left[\frac{\alpha}{\alpha-1}\right]\right) A_{1} e^{A_{1}}
$$


it is easy to see, for every $t \in[0,1]$,

$$
\begin{aligned}
\|v(t)\| & \leq \sum_{k \geq 0} \frac{C_{1}^{k}}{\left[k-\frac{k}{\alpha}\right] !} t^{k\left(1-\frac{1}{\alpha}\right)}\left\|\partial_{x} f\right\| \\
& \leq\left\|\partial_{x} f\right\|+\sum_{k \geq 1} \frac{A_{1}^{\left[k\left(1-\frac{1}{\alpha}\right)\right]+1}}{\left[k-\frac{k}{\alpha}\right] !} t^{\left[k\left(1-\frac{1}{\alpha}\right)\right]}\left\|\partial_{x} f\right\| \\
& \leq\left\|\partial_{x} f\right\|+\left(1+\left[\frac{\alpha}{\alpha-1}\right]\right) A_{1} e^{A_{1} t}\left\|\partial_{x} f\right\|
\end{aligned}
$$

and

$$
\left\|\partial_{x} u(t)\right\|=e^{-t}\left\|\partial_{x} v(t)\right\| \leq e^{-t}\left\{1+\left(1+\left[\frac{\alpha}{\alpha-1}\right]\right) A_{1} e^{A_{1} t}\right\}\left\|\partial_{x} f\right\| .
$$

Moreover, one can apply the above argument on $1 \leq t \leq 2, \ldots, n \leq t \leq n+1, \ldots$, obtaining

$$
\begin{aligned}
\left\|\partial_{x} u(t)\right\| & \leq e^{-(t-1)}\left\{1+\left(1+\left[\frac{\alpha}{\alpha-1}\right]\right) A_{1} e^{A_{1}(t-1)}\right\}\left\|\partial_{x} u(1)\right\| \\
& \leq e^{-(t-1)}\left\{1+\left(1+\left[\frac{\alpha}{\alpha-1}\right]\right) A_{1} e^{A_{1}(t-1)}\right\} e^{-1} A\left\|\partial_{x} f\right\| \quad(1 \leq t \leq 2),
\end{aligned}
$$

and for every $t \in[n, n+1](n \in \mathbf{N})$,

$$
\begin{aligned}
\left\|\partial_{x} u(t)\right\| & \leq e^{-(t-n)}\left\{1+\left(1+\left[\frac{\alpha}{\alpha-1}\right]\right) A_{1} e^{A_{1}(t-n)}\right\}\left(e^{-1} A\right)^{n}\left\|\partial_{x} f\right\| \\
& \leq e^{-t} A^{t+1}\left\|\partial_{x} f\right\| .
\end{aligned}
$$

Let us prove (2.3.10). Noticing the fact $C_{1} \leq 1$ and (2.3.16), it is easy to see, on $0 \leq t \leq 1$,

$$
\begin{aligned}
\|v(t)\| & \leq\left\|\partial_{x} f\right\|+C_{1} \sum_{k \geq 1} \frac{C_{1}^{\left[k-\frac{k}{\alpha}\right]}}{\left[k-\frac{k}{\alpha}\right] !} t^{\left[k-\frac{k}{\alpha}\right]}\left\|\partial_{x} f\right\| \\
& \leq\left\|\partial_{x} f\right\|+C_{1}\left(1+\left[\frac{\alpha}{\alpha-1}\right]\right) e^{C_{1} t}\left\|\partial_{x} f\right\| \\
& =\left\|\partial_{x} f\right\|+A_{2} e^{C_{1} t}\left\|\partial_{x} f\right\|\left(\text { where } A_{2}=C_{1}\left(1+\left[\frac{\alpha}{\alpha-1}\right]\right)\right),
\end{aligned}
$$

and

$$
\left\|\partial_{x} u(t)\right\|=e^{-t}\|v(t)\| \leq e^{-t}\left(1+A_{2} e^{C_{1} t}\right)\left\|\partial_{x} f\right\| \quad(\forall 0 \leq t \leq 1) .
$$

By the same argument, inductively, one has

$$
\begin{aligned}
\left\|\partial_{x} u(t)\right\| & \leq e^{-(t-n)}\left(1+A_{2} e^{C_{1}(t-n)}\right) e^{-n}\left(1+A_{2} e^{C_{1}}\right)^{n}\left\|\partial_{x} f\right\| \\
& \leq e^{-t}\left(1+A_{2} e^{C_{1}}\right)\left(1+A_{2} e^{C_{1}}\right)^{n}\left\|\partial_{x} f\right\| \\
& \left.\leq e^{-t} e^{C_{2}} e^{C_{2} t}\left\|\partial_{x} f\right\| \quad \text { where } C_{2}=\log \left(1+A_{2} e^{C_{1}}\right)\right) \\
& =e^{C_{2}} e^{-\left(1-C_{2}\right) t}\left\|\partial_{x} f\right\|
\end{aligned}
$$

on every $n \leq t \leq n+1(n \in \mathbf{N})$.

Lemma 2.3.4. (Hille-Yosida Theorem for Markov preoperator [8])

The operator $\left(\mathcal{L}_{1}, C_{0}^{\infty}(\mathbf{R})\right)$ satisfies the following three conditions: 
- $C_{0}^{\infty}(\mathbf{R})$ is dense in $C_{\infty}(\mathbf{R})$.

- $\mathcal{L}_{1}$ satisfies maximum principle on $C_{0}^{\infty}(\mathbf{R})$.

- $\forall f \in C_{0}^{\infty}(\mathbf{R}), \exists g \in C_{\infty}(\mathbf{R})$ solving the the equation $\left(\lambda-\mathcal{L}_{1}\right) g=f$.

Then $\left(\mathcal{L}_{1}, C_{0}^{\infty}(\mathbf{R})\right)$ is closable in $C_{\infty}(\mathbf{R})$ and there exists a Markov semigroup $P_{t}(t \geq 0)$ generated by its closure $\left(\mathcal{L}_{1}, \operatorname{Dom}\left(\mathcal{L}_{1}\right)\right)$.

Proof. It is well known that the three conditions in the lemma implies the closability of the infinitesimal generator $([8])$. We only need to check them for $\mathcal{L}_{1}$. The first condition is obvious. $\forall h \in C_{0}^{\infty}(\mathbf{R})$, suppose there exists some $x_{0}$ such that $h\left(x_{0}\right)=\min _{x \in \mathbf{R}} h(x)$.

$$
\left(\partial_{x}^{\alpha} h\right)\left(x_{0}\right)=\lim _{\varepsilon \downarrow 0} \frac{1}{C_{\alpha}} \int_{\left\{\left|y-x_{0}\right|>\varepsilon\right\}} \frac{h(y)-h\left(x_{0}\right)}{\left|y-x_{0}\right|^{\alpha+1}} d y \geq 0
$$

since $h(y)-h\left(x_{0}\right) \geq 0$ for all $y \in \mathbf{R}$. Define $g=\int_{0}^{\infty} e^{-\lambda t} u(t) d t$, where $u(t)$ is the classical solution of (2.2.1) with initial data $f . g$ uniformly converges if $\lambda>\lambda_{0}$ and $\lambda_{0}>0$ is large enough, since

$$
\|u(t)\| \leq\|f\|+\|b\| \int_{0}^{t} e^{-s} A^{s+1} d s\left\|\partial_{x} f\right\|=\|f\|+\|b\| \frac{\left(\left(\frac{A}{e}\right)^{t}-1\right) A}{\log \frac{A}{e}}\left\|\partial_{x} f\right\|
$$

from (2.3.9) and (2.2.2). Hence

$$
\mathcal{L}_{1} g=\int_{0}^{\infty} e^{-\lambda t} \mathcal{L}_{1} u(t) d t=\int_{0}^{\infty} e^{-\lambda t} \partial_{t} u(t) d t=-f+\lambda g .
$$

Moreover, it is obvious to have $g \in C_{\infty}(\mathbf{R})$ since $u(t) \in C_{\infty}(\mathbf{R})$.

Lemma 2.3.5. (Ergodicity for $S_{t}$ ) If $f \in C_{\infty}^{1}(\mathbf{R})$, then

$$
\lim _{t \rightarrow \infty} S_{t} f(x)=c \quad \forall x \in \mathbf{R}
$$

where $c$ is a constant independent of $x$.

Proof. $\forall t_{2}>t_{1}>0$, we have, with some $A>0$,

$$
\begin{aligned}
\left|S_{t_{2}} f(0)-S_{t_{1}} f(0)\right| & \leq\left[\int_{y:|y| \leq A}\left|p\left(\frac{1-e^{-\alpha t_{2}}}{\alpha} ; 0, y\right)-p\left(\frac{1-e^{-\alpha t_{1}}}{\alpha} ; 0, y\right)\right| d y\right. \\
& \left.+\int_{y:|y| \geq A} p\left(\frac{1-e^{-\alpha t_{2}}}{\alpha} ; 0, y\right) d y+\int_{y:|y| \geq A} p\left(\frac{1-e^{-\alpha t_{1}}}{\alpha} ; 0, y\right) d y\right] \cdot|| f \mid \| \\
& =\left[I_{1}+I_{2}+I_{3}\right] \cdot\|f\| .
\end{aligned}
$$


For any fixed $A$, by Lebesgue dominated convergence theorem,

$$
I_{1} \rightarrow 0, \quad \text { as } t_{1}, t_{2} \rightarrow \infty
$$

According to the heat kernel estimate (2.1.12), $\forall t>1$,

$$
\int_{y:|y| \geq A} p\left(\frac{1-e^{-\alpha t_{1}}}{\alpha} ; 0, y\right) d y \leq \int_{y:|y| \geq A} \frac{K}{|y|^{\alpha+1}} d y \rightarrow 0, \quad \text { as } A \rightarrow \infty
$$

Combine (2.3.29) and (2.3.30), we obtain

$$
c:=\lim _{t \rightarrow \infty} S_{t} f(0)
$$

$\forall x \in \mathbf{R}$, one has

$$
\begin{aligned}
\left|S_{t} f(x)-c\right| & \leq\left|S_{t} f(x)-S_{t} f(0)\right|+\left|S_{t} f(0)-c\right| \leq\left|\int_{0}^{x} \partial_{z} S_{t} f(z) d z\right|+\left|S_{t} f(0)-c\right| \\
& \stackrel{(2.1 .15)}{\leq}\left|S_{t} f(0)-c\right|+|x| \cdot e^{-t}|| \partial_{x} f|| \rightarrow 0 \quad(t \rightarrow \infty) .
\end{aligned}
$$

\subsection{Proof of Theorems I, II and Corollary 2.2.1}

Proof of Theorem I: According to the classical semigroup theory, $\forall f \in C_{\infty}^{2}(\mathbf{R}) \subset$ $\operatorname{Dom}\left(\mathcal{L}_{1}\right), P_{t} f$ uniquely solves (2.2.1). From Lemma 2.3.1, we define another semigroup $\tilde{P}_{t}(t \geq 0)$ by

$$
\tilde{P}_{t} f=u(t) \quad \forall f \in C_{\infty}^{2}(\mathbf{R}) .
$$

By the uniqueness of the solution, it is obvious that

$$
P_{t} f=\tilde{P}_{t} f \quad \forall f \in C_{\infty}^{2}(\mathbf{R})
$$

Since $C_{\infty}^{2}(\mathbf{R})$ is dense in $C_{\infty}(\mathbf{R})$ under uniform norm, we can extend $(2.4 .1)$ to $C_{\infty}(\mathbf{R})$, i.e.

$$
P_{t} f=\tilde{P}_{t} f \quad \forall f \in C_{\infty}(\mathbf{R})
$$

Proof of Theorem II: We prove the theorem in the case of $f \in C_{\infty}^{1}(\mathbf{R})$, and the case of $f \in C_{\infty}(\mathbf{R})$ can be done in a classical but simple approximate procedure. $\forall t_{2} \geq t_{1}>0$, it is easy to see

$$
\left|P_{t_{2}} f(0)-P_{t_{1}} f(0)\right| \leq\left|S_{t_{2}} f(0)-S_{t_{1}} f(0)\right|+\left|\int_{0}^{t_{2}} S_{t_{2}-s}\left[b \partial_{x} P_{s} f\right](0) d s-\int_{0}^{t_{1}} S_{t_{1}-s}\left[b \partial_{x} P_{s} f\right](0) d s\right|
$$


For the second term on the right hand side of (2.4.3), one has, with some $B>0$

$$
\begin{aligned}
& \quad \int_{0}^{t_{2}} S_{t_{2}-s}\left[b \partial_{x} P_{s} f\right](0) d s-\int_{0}^{t_{1}} S_{t_{1}-s}\left[b \partial_{x} P_{s} f\right](0) d s \mid \\
& \leq \int_{0}^{B}\left|S_{t_{2}-s}\left[b \partial_{x} P_{s} f\right](0) d s-S_{t_{1}-s}\left[b \partial_{x} P_{s} f\right](0)\right| d s+\left|\int_{B}^{t_{2}} S_{t_{2}-s}\left[b \partial_{x} P_{s} f\right](0) d s\right| \\
& +\left|\int_{B}^{t_{1}} S_{t_{1}-s}\left[b \partial_{x} P_{s} f\right](0) d s\right| \\
& =I_{1}+I_{2}+I_{3}
\end{aligned}
$$

By (2.3.9) and (2.3.28), for any fixed $B>0$, we have

$$
I_{1} \rightarrow 0, \quad \text { as } t_{1}, t_{2} \rightarrow \infty
$$

For $I_{2}$ ( $I_{3}$ can be treated by the same arguments), as $t_{2}, B \rightarrow \infty$,

$$
\begin{aligned}
& I_{2} \leq \int_{B}^{t_{2}}\left\|S_{t_{2}-s}\left[b \partial_{x} P_{s} f\right]\right\| d s \leq \int_{B}^{t_{2}}\|b\| \cdot\left\|\partial_{x} P_{s} f\right\| d s \quad \text { (St is contraction) } \\
& \stackrel{(2.3 .10)}{\leq} \int_{B}^{t_{2}}\|b\| e^{C_{2}} e^{-\left(1-C_{2}\right) s}\left\|\partial_{x} f\right\| d s \rightarrow 0
\end{aligned}
$$

Hence, from lemma 2.3.5 and the above estimates on $I_{1}, I_{2}, I_{3}$, it is obvious to have

$$
\lim _{t \rightarrow \infty} P_{t} f(0):=c \quad(\text { a constant }) .
$$

$\forall x \in \mathbf{R}$, using the similar argument for obtaining (2.3.32) and (2.3.10), we have

$$
\lim _{t \rightarrow \infty} P_{t} f(x)=c .
$$

Proof of Corollary 2.2.1: It is easy to check that the solution to (1.0.1) satisfies

$$
X_{t}=e^{-t} x-\int_{0}^{t} e^{-(t-s)} d Z_{s}+\int_{0}^{t} e^{-(t-s)} b\left(X_{s}\right) d s
$$

which implies

$$
\begin{aligned}
E\left|X_{t}\right| & \leq|x|+E\left|\int_{0}^{t} e^{t-s} d Z_{s}\right|+|| b \|_{\infty}\left(1-e^{-t}\right) \\
& \leq|x|+E\left|Z_{1}\right| \cdot \int_{0}^{t} e^{-(t-s)} s^{1 / \alpha-1} d s+\|b\|_{\infty}
\end{aligned}
$$


since $\alpha$-stable process is a stationary process with independent increments and $E \mid Z_{t}-$ $Z_{s}|=| t-\left.s\right|^{1 / \alpha} E\left|Z_{1}\right|$, and thus

$$
\sup _{0 \leq t<\infty} E\left|X_{t}\right| \leq|x|+C+\|b\|_{\infty}
$$

where $C>0$ is some constant independent of $x$. Hence, the transition probability family of the system $\{p(t ; x, d y)\}_{t \geq 0}$ is tight, according to Prohorov theorem, there exists a measure $\mu_{x}$ depending on $x$ and subsequence $\left\{t_{k}\right\}_{k \in \mathbf{N}}$ with $t_{k} \rightarrow \infty$ such that

$$
p\left(t_{k} ; x, d y\right) \rightarrow \mu_{x} \text { weakly. }
$$

Noticing Theorem II, for any given $\phi \in C_{\infty}(\mathbf{R})$, we have

$$
\left|P_{t} \phi(x)-\mu_{x}(\phi)\right| \leq\left|P_{t} \phi(x)-P_{t_{n}} \phi(x)\right|+\left|P_{t_{n}} \phi(x)-\mu_{x}(\phi)\right| \rightarrow 0, \text { as } t, t_{n} \rightarrow \infty,
$$

i.e. $p(t ; x, d y) \rightarrow \mu_{x}$ as $t \rightarrow \infty$, which also easily implies that $\mu_{x}$ is an invariant measure of the system.

Denote $\mathcal{I}$ as the set of the all invariant measures for (1.0.1). Given any two invariant measures $\mu_{1}, \mu_{2} \in \mathcal{I}$, then for every $\phi \in C_{\infty}(\mathbf{R})$,

$$
\begin{aligned}
\left|\mu_{1}(\phi)-\mu_{2}(\phi)\right| & =\lim _{t \rightarrow \infty}\left|\iint \phi(z) p(t ; x, d z) \mu_{1}(d x)-\iint \phi(z) p(t ; y, d z) \mu_{2}(d y)\right| \\
& \leq \lim _{t \rightarrow \infty} \int\left|\int \phi(z) p(t ; x, d z)-\phi(z) p(t ; y, d z)\right| \mu_{1}(d x) \mu_{2}(d y) \\
& =\int \lim _{t \rightarrow \infty}\left|\int \phi(z) p(t ; x, d z)-\phi(z) p(t ; y, d z)\right| \mu_{1}(d x) \mu_{2}(d y) \\
& =0 . \quad \text { (noticing Theorem II) }
\end{aligned}
$$

So $\mu_{1}=\mu_{2}$, which means that $\mathcal{I}$ only includes one element.

As for the strong mixing property of (1.0.2), according to Corollary 3.4.3 in [6], the above convergence of the transition probabilities implies the system (1.0.1) is strongly mixing.

\section{$3 \quad$ Infinite Dimensional Interacting $\alpha$-stable Systems}

We will only study our system in the configuration space of $(\mathbf{R})^{\mathbf{Z}^{d}}$, but our approaches and results are also true for $\left(\mathbf{R}^{\mathbf{n}}\right)^{\mathbf{Z}^{d}}$. We first list the notations as follows, which will be frequently used in this section.

- Configuration Space $\Omega: \Omega=\mathbf{R}^{\mathbf{Z}^{d}} . \forall x \in \Omega, x=\left(x_{i}\right)_{i \in \mathbf{Z}^{d}}, x_{i} \in \mathbf{R} ; \forall \Lambda \subset \mathbf{Z}^{d}, x_{\Lambda}=$ $\left(x_{i}\right)_{i \in \Lambda}$.

- Lattice $\Gamma: \Gamma=\mathbf{Z}^{d} . \exists\left\{\Gamma_{N} ; N \in \mathbf{N}\right\}$ such that $\Gamma_{N} \subset \subset \mathbf{Z}^{d}$ and $\lim _{N \uparrow \infty} \Gamma_{N}=\mathbf{Z}^{d}$. Given a cube $\Lambda \subset \subset \mathbf{Z}^{d}$ centred at $0, \Lambda_{i}:=\{i+j ; j \in \Lambda\}$ for $i \in \mathbf{Z}^{d}$ and $\Gamma_{N}^{\Lambda}=\{i \in$ $\left.\mathbf{Z}^{d} ; \operatorname{dist}\left(i, \Gamma_{N}\right)<\operatorname{diam}(\Lambda)\right\}$ where $\operatorname{dist}(i, j)=\sum_{1 \leq k \leq d}\left|i_{k}-j_{k}\right| \forall i, j \in \mathbf{Z}^{d}$

- Local Functions Spaces $\mathcal{D}$ : For any $\Lambda \subset \subset \mathbf{Z}^{d}, \mathcal{D}_{\Lambda}=\{f: f$ is a bounded continuous function depending on the configurations in $\Lambda$ and $f$ vanishes at $\infty$. . $\mathcal{D}=\bigcup_{\Lambda \subset \subset \mathbf{Z}^{d}} \mathcal{D}_{\Lambda} . \mathcal{D}^{k}=\left\{f \in \mathcal{D} ; f\right.$ is $\left.C^{k}\right\}$. We use $\Lambda(f)$ to denote the localization set of the local function $f$, i.e. the smallest set $\Lambda^{\prime} \subset \mathbf{Z}^{d}$ such that $f \in \mathcal{D}_{\Lambda^{\prime}}$. 
- Potential $\mathcal{U}$ : In this paper, $\mathcal{U}=\left\{U_{\Lambda_{i}} \in \mathcal{D}_{\Lambda_{i}} ; i \in \mathbf{Z}^{d}\right\}$. A typical example for $\mathcal{U}$ is $U_{\Lambda_{i}}=\sum_{X \ni i} \phi_{X}$ where $\phi_{X} \in \mathcal{V}=\{\phi ; \phi$ is local function such that $\operatorname{diam}(\Lambda(\phi))<R\}$ where $R<\infty$ is some constant and $\operatorname{diam}(\Lambda)=\max \{d(i, j): i, j \in \Lambda\}$.

- Infinite Dimensional Infinitesimal Generator: $\mathcal{L}=\sum_{i \in \mathbf{Z}^{d}}\left[\partial_{i}^{\alpha}-x_{i} \partial_{i}\right]+\sum_{i \in \mathbf{Z}^{d}} U_{\Lambda_{i}}(x) \partial_{i}$ where $\partial_{i}=\partial_{x_{i}}$ and $\partial_{i}^{\alpha}=\partial_{x_{i}}^{\alpha}$. We also simply denote $\partial_{i j}=\partial_{x_{i}} \partial_{x_{j}}$. For simplicity, we drop $\Lambda$ in the potentials and write $U_{i}=U_{\Lambda_{i}}$. We will approximate $\mathcal{L}$ by the operators as follows:

$$
\mathcal{L}_{N}=\sum_{i \in \mathbf{Z}^{d}}\left[\partial_{i}^{\alpha}-x_{i} \partial_{i}\right]+\sum_{i \in \Gamma_{N}} U_{i}(x) \partial_{i}
$$

Note that $U_{i}$ is a function depending on $x_{\Lambda_{i}}$, not just $x_{i}$.

- Semigroups: $\left\{S_{t}\right\}_{t \geq 0}$ is the semigroup generated by product Ornstein-Uhlenbeck $\alpha$-stable operator

$$
\sum_{i \in \mathbf{Z}^{d}}\left[\partial_{i}^{\alpha}-x_{i} \partial_{i}\right]
$$

$\left\{P_{t}^{N}\right\}_{t \geq 0}$ and $\left\{P_{t}\right\}_{t \geq 0}$ are the semigroup generated by $\mathcal{L}_{N}$ and $\mathcal{L}$ respectively.

- Norms: ||.|| in the following context is the uniform norm. The |||.||| is defined by

$$
\|f\|\left\|=\sum_{i \in \mathbf{Z}^{d}}\right\| \partial_{i} f \|, \quad \forall f \in \mathcal{D}^{1} .
$$

Formally the Kolmogorov backward equation of the system (1.0.2) is

$$
\left\{\begin{array}{l}
\partial_{t} u=\sum_{i \in \mathbf{Z}^{d}}\left[\partial_{i}^{\alpha}-x_{i} \partial_{i}\right] u+\sum_{i \in \mathbf{Z}^{d}} U_{\Lambda_{i}}(x) \partial_{i} u \\
u(0)=f
\end{array}\right.
$$

which is an infinite dimensional equation and hard to be solved directly. Alternatively, we consider the infinitesimal generator $\mathcal{L}$ from which a Markov semigroup may be constructed. One can understand the properties of the systems (1.0.2) and (3.0.9) by studying the Markov semigroup. Because we can extend the conclusions about the semigroup on $\mathcal{D}^{\infty}$ to $C_{\infty}(\Omega)$ by the fact that $\mathcal{D}^{\infty}$ is dense in $C_{\infty}(\Omega)$ under uniform norm, we only construct the semigroup and prove its ergodic property on $\mathcal{D}^{\infty}$. The main results of this section are the following two theorems.

Theorem III. If $\sup _{i}\left\|\left|U_{i}\right|\right\|+\sup _{i}\left\|U_{i}\right\|<\infty$, then $\forall f \in \mathcal{D}^{\infty}$, we have

$$
\lim _{N \rightarrow \infty} P_{t}^{N} f=P_{t} f \quad \text { under uniform norm. }
$$

Theorem IV. Suppose that

$$
\gamma:=\sum_{i \in \mathbf{Z}^{d}}\left\|\partial_{i} U_{i}\right\|, \beta:=\hat{C} \sum_{i \in \mathbf{Z}^{d}}\left\|U_{i}\right\|, \eta:=\sup _{j}\left\|U_{j}\right\|
$$


are all finite, where $\hat{C}$ is the constant in lemma 2.1.2. Set

$$
|i|=\sum_{k=1}^{d}\left|i_{k}\right|\left(i \in \mathbf{Z}^{d}\right), B_{R, \rho}=\left\{x:\left|x_{i}\right| \leq R|i|^{\rho}\right\} \quad(R>0, \rho>0), \quad \mathbf{B}=\bigcup_{R>0, \rho>0} B_{R, \rho} .
$$

If

$$
\frac{\alpha \beta}{\alpha-1}<e^{-(\eta+\gamma)}, \quad 1-\eta-\gamma-\theta>0
$$

with $\theta=\log \left(1+\frac{\alpha \beta e^{\eta+\gamma}}{\alpha-1-\alpha \beta e^{\eta+\gamma}}\right)$, then $\forall x \in \mathbf{B}$, we have

$$
\lim _{t \rightarrow \infty} P_{t} f(x)=a
$$

where $a$ is some constant independent of $x$.

\subsection{Proof of Theorem III}

Lemma 3.1.1. For any $\mathcal{L}_{N}$, there exists a Markov semigroup $P_{t}^{N}$ satisfying

$$
\partial_{t} P_{t}^{N} f=\mathcal{L}_{N} P_{t}^{N} f
$$

where $f \in \mathcal{D}^{\infty}$ such that $\Lambda(f) \subset \Gamma_{N}$. Moreover,

$$
P_{t}^{N} f=S_{t} f+\int_{0}^{t} S_{t-s} \sum_{i \in \Gamma_{N}} U_{i} \partial_{i} P_{s}^{N} f d s
$$

where $S_{t}$ is the trivial product semigroup generated by $\sum_{i \in \mathbf{Z}^{d}}\left(\partial_{i}^{\alpha}-x_{i} \partial_{i}\right)$.

Proof. By the same argument as proving Theorem I.

Proof of Theorem III: The proof uses the similar arguments as in chapter 8 of [7]. It is sufficient to check that $\left\{P_{t}^{N}\right\}_{N}$ is a Cauchy sequence under uniform norm. $\forall \Gamma_{M} \supset$ $\Gamma_{N} \supset \Lambda(f)$, it is easy to check

$$
\begin{aligned}
\left\|P_{t}^{M} f-P_{t}^{N} f\right\| & \leq\left\|\int_{0}^{t} \frac{d}{d s} P_{t-s}^{M} P_{s}^{N} f d s\right\| \leq\left\|\int_{0}^{t} P_{t-s}^{M}\left(\mathcal{L}_{M}-\mathcal{L}_{N}\right) P_{s}^{N} f d s\right\| \\
& \leq \int_{0}^{t}\left\|\left(\mathcal{L}_{M}-\mathcal{L}_{N}\right) P_{s}^{N} f\right\| d s \leq \int_{0}^{t} \sum_{\Gamma_{M} \backslash \Gamma_{N}}\left\|U_{i}\right\| \cdot\left\|\partial_{i} P_{s}^{N} f\right\| d s
\end{aligned}
$$

By the easy fact $\frac{d}{d s} P_{t-s}^{N} \partial_{i} P_{s}^{N} f=P_{t-s}^{N}\left[\partial_{i}, \mathcal{L}_{N}\right] P_{s}^{N} f\left(\right.$ where $\left[\partial_{i}, \mathcal{L}_{N}\right]=\partial_{i} \mathcal{L}_{N}-\mathcal{L}_{N} \partial_{i}=$ $\left.-\sum_{j \in \mathbf{Z}^{d}} \delta_{i j} \partial_{j}+\sum_{j \in \Gamma_{N}} \partial_{i} U_{j} \partial_{j} f\right)$ and Markov property of $P_{t}^{N}$, we have

$$
\begin{aligned}
\left\|\partial_{i} P_{t}^{N} f\right\| & \leq\left\|\partial_{i} f\right\|+\int_{0}^{t}\left\|\left[\partial_{i}, \mathcal{L}_{N}\right] P_{s}^{N} f\right\| d s \\
& \leq\left\|\partial_{i} f\right\|+\int_{0}^{t} \sum_{j \in \mathbf{Z}^{d}}\left(\delta_{i j}+\left\|\partial_{i} U_{j}\right\|\right) \cdot\left\|\partial_{j} P_{s}^{N} f\right\| d s .
\end{aligned}
$$


Denote $c_{i j}=\left\|\partial_{i} U_{j}\right\|$ and $e_{i j}(t)=\sum_{n \geq 0} \frac{t^{n}}{n !}(c+\delta)_{i j}^{n}$, then $c$ and $e(t)$ are both operators in $l_{1}$ with norm

$$
\|c\|_{l_{1}} \leq \sup _{j}\left\|\left|U_{j}\right|\right\|, \quad\|e(t)\|_{l_{1}} \leq \exp \left\{\left(\|c\|_{l_{1}}+1\right) t\right\} \leq \exp \left\{\left(\sup _{j}\left\|\left|U_{j} \|\right|+1\right) t\right\}\right.
$$

(Indeed, $\left.\left|\sum_{i \in \mathbf{Z}^{d}} \sum_{j \in \mathbf{Z}^{d}} e_{i j}(t) \alpha_{j}\right| \leq \sum_{n \geq 0} \frac{t^{n}}{n !}\left(|| c \mid l_{l_{1}}+1\right)^{n} \sum_{j}\left|\alpha_{j}\right| \leq \exp \left\{\left(\sup _{j}||\left|U_{j}\right|||+1\right) t\right\} \sum_{j}\left|\alpha_{j}\right|.\right)$ Iterating (3.1.4), we have

$$
\begin{aligned}
& \left\|\partial_{i} P_{t}^{N} f\right\| \leq \sum_{j \in \mathbf{Z}^{d}} e_{i j}(t)\left\|\partial_{j} f\right\| \\
& \|\| P_{t}^{N} f\left|\left\|\leq \exp \left\{\left(\|c\|_{l_{1}}+1\right) t\right\}\right\|\|f\|\right| \leq \exp \left\{\left(\sup _{j}\left\|\left|U_{j} \|\right|+1\right) t\right\}\|\| f\|\|,\right.
\end{aligned}
$$

and have by noticing (3.1.5)

$$
\begin{aligned}
& \left\|P_{t}^{M} f-P_{t}^{N} f\right\| \leq \int_{0}^{t} \sum_{i \in \Gamma_{M} \backslash \Gamma_{N}}\left\|U_{i}\right\| \sum_{j \in \mathbf{Z}^{d}} e_{i j}(t)\left\|\partial_{j} f\right\| d s \\
& \leq \sup _{i}\left\|U_{i}\right\| \int_{0}^{t} \sum_{i \in \Gamma_{M} \backslash \Gamma_{N}} \sum_{j \in \mathbf{Z}^{d}} e_{i j}(t)\left\|\partial_{j} f\right\| d s \rightarrow 0 \quad(N, M \rightarrow \infty)
\end{aligned}
$$

\subsection{Proof of Theorem IV}

\section{Lemma 3.2.1. (Finite Speed of Propagation)}

Given any approximate semigroup $P_{t}^{N}$ and $f \in \mathcal{D}^{\infty}, \forall i \notin \Lambda(f)$, then

$$
\left\|\partial_{i} P_{t}^{N} f\right\| \leq \frac{t^{N_{i}}(1+\eta)^{N_{i}}}{N_{i} !} e^{(\eta+1) t} \mid\|f\| \|
$$

where $N_{i}=\left[\frac{\operatorname{dist}(i, \Lambda(f))}{\operatorname{diam}(\Lambda)}\right]$ and $\eta$ is the same as in Theorem IV. Moreover, for any $A>0$, there exists some $B \geq 1$ such that, when $N_{i}>B t$, we have

$$
\left\|\partial_{i} P_{t}^{N} f\right\| \leq e^{-A t-A N_{i}}|| f \mid \|
$$

Proof. The arguments are similar to those of [7] (pp 88-90). Recall the equation (3.1.4)

$$
\begin{aligned}
\left\|\partial_{i} P_{t}^{N} f\right\| & \leq\left\|\partial_{i} f\right\|+\int_{0}^{t} \sum_{j \in \mathbf{Z}^{d}}\left(\delta_{i j}+\left\|\partial_{i} U_{j}\right\|\right) \cdot\left\|\partial_{j} P_{s}^{N} f\right\| d s \\
& \leq \sum_{n=0}^{N_{i}-1} \frac{t^{n}}{n !}(c+\delta)_{i j}^{n}\left\|\partial_{j} f\right\|+\sum_{n=N_{i}}^{\infty} \frac{t^{n}}{n !}(c+\delta)_{i j}^{n}\left\|\partial_{j} f\right\| .
\end{aligned}
$$

Since $\Lambda\left(U_{i}\right)=\Lambda_{i}$, one can check that $([7]$, pp 90)

$$
\sum_{n=0}^{N_{i}-1} \frac{t^{n}}{n !}(c+\delta)_{i j}^{n}\left\|\partial_{j} f\right\|=0,
$$


thus

$$
\left\|\partial_{i} P_{t}^{N} f\right\| \leq \sum_{n=N_{i}}^{\infty} \frac{t^{n}}{n !}(c+\delta)_{i j}^{n}\left\|\partial_{j} f\right\| \leq \frac{t^{N_{i}}(1+\eta)^{N_{i}}}{N_{i} !} e^{(1+\eta) t}\|\| f\|\|
$$

where $\eta=\|c\|_{l_{1}}=\sup _{j}\left\|\left|U_{j}\right|\right\|$. Choosing $B \geq 1$ such that

$$
2-\log B+\log (1+\eta)+\frac{1+\eta}{B} \leq-2 A,
$$

as $n>B t$, one has

$$
\begin{aligned}
& \frac{t^{n}(1+\eta)^{n}}{n !} e^{(1+\eta) t} \leq \exp \{n \log t(1+\eta)-n \log n+2 n+(1+\eta) t\} \\
& \leq \exp \left\{n \log \frac{1+\eta}{B}+2 n+(1+\eta) \frac{n}{B}\right\} \leq \exp \{-2 A n\} \leq \exp \{-A n-A t\} .
\end{aligned}
$$

Replacing $n$ by $N_{i}$, we conclude the proof.

Lemma 3.2.2. Let $\gamma, \beta, \eta$ and $\theta$ be the same as in Theorem IV. If $\frac{\alpha \beta}{\alpha-1}<e^{-(\eta+\gamma)}$, then $\forall f \in \mathcal{D}^{\infty}$, we have

$$
\left\|| | P _ { t } ^ { N } f \left|\left\|\leq e^{-(1-\eta-\gamma-\theta) t}|\|f \mid\|, \quad \forall N .\right.\right.\right.
$$

Proof. Noticing the fact $\Lambda\left(P_{t}^{N} f\right)=\Gamma_{N}^{\Lambda}$ (because the interaction range of every $U_{i}$ is $\operatorname{diam}(\Lambda))$, and using integration by part formula and the fact $\partial_{y} p\left(\frac{1-e^{-\alpha t}}{\alpha} ; e^{-t} x, y\right)=$ $e^{t} \partial_{x} p\left(\frac{1-e^{-\alpha t}}{\alpha} ; e^{-t} x, y\right)$ (see $\mathrm{p}(\mathrm{t} ; \mathrm{x}, \mathrm{y})$ in Lemma 2.1.1), one has

$$
\begin{aligned}
& S_{t-s}\left(U_{j} \partial_{i j} P_{s}^{N} f\right)(x) \stackrel{\Lambda\left(P_{t}^{N} f\right)=\Gamma_{N}^{\Lambda}}{=} \int \prod_{k \in \Gamma_{N}^{\Lambda}} p\left(\frac{1-e^{-\alpha(t-s)}}{\alpha} ; e^{-(t-s)} x_{k}, y_{k}\right) U_{j}\left(y_{\Lambda_{j}}\right) \partial_{i j} P_{s}^{N} f(y) d y_{\Gamma_{N}^{\Lambda}} \\
&=-\int \partial_{y_{j}} \prod_{k \in \Gamma_{N}^{\Lambda}} p\left(\frac{1-e^{-\alpha(t-s)}}{\alpha} ; e^{-(t-s)} x_{k}, y_{k}\right) U_{j}\left(y_{\Lambda_{j}}\right) \partial_{i} P_{s}^{N} f(y) d y_{\Gamma_{N}^{\Lambda}} \\
&-\int \prod_{k \in \Gamma_{N}^{\Lambda}} p\left(\frac{1-e^{-\alpha(t-s)}}{\alpha} ; e^{-(t-s)} x_{k}, y_{k}\right) \partial_{j} U_{j}\left(y_{\Lambda_{j}}\right) \partial_{i} P_{s}^{N} f(y) d y_{\Gamma_{N}^{\Lambda}} \\
&=-e^{t-s} \partial_{x_{j}} \int \prod_{k \in \Gamma_{N}^{\Lambda}} p\left(\frac{1-e^{-\alpha(t-s)}}{\alpha} ; e^{-(t-s)} x_{k}, y_{k}\right) U_{j}\left(y_{\Lambda_{j}}\right) \partial_{i} P_{s}^{N} f(y) d y_{\Gamma_{N}^{\Lambda}} \\
&-\int \prod_{k \in \Gamma_{N}^{\Lambda}} p\left(\frac{\left.1-e^{-\alpha(t-s)} ; e^{-(t-s)} x_{k}, y_{k}\right) \partial_{j} U_{j}\left(y_{\Lambda_{j}}\right) \partial_{i} P_{s}^{N} f(y) d y_{\Gamma_{N}}}{\alpha}=-e^{t-s} \partial_{j} S_{t-s}\left(U_{j} \partial_{i} P_{s}^{N} f\right)-S_{t-s}\left(\partial_{j} U_{j} \partial_{i} P_{s}^{N} f\right) .\right.
\end{aligned}
$$


By (3.1.2), (2.1.15), (3.2.7) and (2.1.16), we have, for all $i \in \mathbf{Z}^{d}$,

$$
\begin{aligned}
& \left\|\partial_{i} P_{t}^{N} f\right\| \leq e^{-t}\left\|\partial_{i} f\right\|+\int_{0}^{t} e^{-(t-s)} \sum_{j \in \Gamma_{N}}\left\|S_{t-s} \partial_{i}\left(U_{j} \partial_{j} P_{s}^{N} f\right)\right\| d s \\
& \leq e^{-t}\left\|\partial_{i} f\right\|+\int_{0}^{t} e^{-(t-s)} \sum_{j \in \Gamma_{N}}\left\|\partial_{i} U_{j}\right\| \cdot\left\|\partial_{j} P_{s}^{N} f\right\| d s+\int_{0}^{t} e^{-(t-s)} \sum_{j \in \Gamma_{N}}\left\|S_{t-s}\left(U_{j} \partial_{i j} P_{s}^{N} f\right)\right\| d s \\
& \stackrel{(3.2 .7)}{\leq} e^{-t}\left\|\partial_{i} f\right\|+\int_{0}^{t} e^{-(t-s)} \sum_{j \in \Gamma_{N}} c_{i j}\left\|\partial_{j} P_{s}^{N} f\right\| d s \\
& +\int_{0}^{t} e^{-(t-s)} \sum_{j \in \Gamma_{N}}\left\|\partial_{j} U_{j}\right\| \cdot\left\|\partial_{i} P_{s}^{N} f\right\| d s+\int_{0}^{t} \sum_{j \in \Gamma_{N}}\left\|\partial_{j} S_{t-s}\left(U_{j} \partial_{i} P_{s}^{N} f\right)\right\| d s \\
& \leq e^{-t}\left\|\partial_{i} f\right\|+\int_{0}^{t} e^{-(t-s)} \sum_{j \in \Gamma_{N}} c_{i j}\left\|\partial_{j} P_{s}^{N} f\right\| d s+\int_{0}^{t} e^{-(t-s)}\left(\gamma+\frac{\beta}{(t-s)^{\frac{1}{\alpha}} \wedge 1}\right)\left\|\partial_{i} P_{s}^{N} f\right\| d s
\end{aligned}
$$

where $c_{i j}=\left\|\partial_{i} U_{j}\right\|$. Hence,

$$
e^{t}\left|\left\|P_{t}^{N} f\right\|\right| \leq\||f|\|+\int_{0}^{t}\left(\eta+\gamma+\frac{\beta}{(t-s)^{\frac{1}{\alpha}} \wedge 1}\right) e^{s}\left|\left\|P_{s}^{N} f \mid\right\| d s .\right.
$$

When $0 \leq t \leq 1$, by (3.1.6) and (3.2.9), we have the following Gronwall's type inequality

$$
\begin{aligned}
e^{t} \mid\left\|P_{t}^{N} f\right\| \| & \leq\||f|\|+\int_{0}^{t}(\eta+\gamma) e^{s}\left\|\left|P_{s}^{N} f\left\|\left|d s+\left(\int_{0}^{t} \frac{\beta}{(t-s)^{\frac{1}{\alpha}}} d s\right) e^{2+\eta}\right|\right\| f\|\|\right.\right. \\
& \leq\left\|| | f \left|\left\|+\int_{0}^{t}(\eta+\gamma) e^{s}||\left|P_{s}^{N} f\left\|\left|d s+\frac{\alpha \beta}{\alpha-1} e^{2+\eta}\right|\right\| f\right|\right\|,\right.\right.
\end{aligned}
$$

and thus

$$
e^{t}||\left|P_{t}^{N} f\right|\left\|\leq e^{(\eta+\gamma) t}\left(1+\frac{\alpha \beta}{\alpha-1} e^{2+\eta}\right)|\||f|\| .\right.
$$

Set

$$
K_{1}=e^{(\eta+\gamma)}\left(1+\frac{\alpha \beta}{\alpha-1} e^{2+\eta}\right),
$$

by the above estimate on $e^{t}\left\|\mid P_{t}^{N} f\right\| \|,(3.2 .9)$ implies

$$
e^{t}||\left|P _ { t } ^ { N } f \left\|\left|\leq\left\|| | f \left|\left\|+\int_{0}^{t}(\eta+\gamma) e^{s}\left|\left\|P_{s}^{N} f\left|\left\|d s+\frac{\alpha \beta}{\alpha-1} K_{1}\right\|\right| f\right\| \|,\right.\right.\right.\right.\right.\right.\right.
$$


and (3.2.11) is improved to be

$$
e^{t}\left\|\left|P_{t}^{N} f\left\|\left|\leq e^{(\eta+\gamma) t}\left(1+\frac{\alpha \beta}{\alpha-1} K_{1}\right)\right|\right\| f\|\| .\right.\right.
$$

By induction, we have

$$
e^{t}\left|\left\|P_{t}^{N} f\right\|\right| \leq e^{(\eta+\gamma) t}\left(1+\frac{\alpha \beta}{\alpha-1} K_{n}\right)\||| f\| \mid \quad n=1,2, \ldots
$$

where $K_{n}=e^{(\eta+\gamma) t}\left(1+\frac{\alpha \beta}{\alpha-1} K_{n-1}\right)$. It is easy to see that if $\frac{\alpha \beta}{\alpha-1}<e^{-(\eta+\gamma)}$,

$$
K:=\lim _{n \rightarrow \infty} K_{n}=\frac{e^{\eta+\gamma}}{1-\frac{\alpha \beta}{\alpha-1} e^{\eta+\gamma}} .
$$

Hence,

$$
\left\|P_{t}^{N} f\right\| \mid \leq e^{-(1-\eta-\gamma) t}\left(1+\frac{\alpha \beta}{\alpha-1} K\right)\|\| f\|\| \quad(0 \leq t \leq 1) .
$$

Using the same method, when $n \leq t \leq n+1$, we have

$$
\left\|\left|P_{t}^{N} f\left\|\left|\leq e^{-(1-\eta-\gamma) t}\left(1+\frac{\alpha \beta}{\alpha-1} K\right)^{n}\right|\right\| f\right|\right\| \leq e^{-(1-\eta-\gamma-\theta) t}|||f| \| .
$$

Lemma 3.2.3. If $1-\eta-\gamma-\theta>0$, then we have some constant a such that

$$
\lim _{t \rightarrow \infty} P_{t} f(0):=a
$$

where $f, \eta, \gamma, \theta$ are the same as those in Lemma 3.2.2.

Proof. For $\forall t_{2}>t_{1}>T$ (with a large number $T$ to be determined later), it is obvious to see

$$
\left|P_{t_{2}} f(0)-P_{t_{1}} f(0)\right| \leq\left|P_{t_{2}} f(0)-P_{t_{2}}^{N} f(0)\right|+\left|P_{t_{2}}^{N} f(0)-P_{t_{1}}^{N} f(0)\right|+\left|P_{t_{1}} f(0)-P_{t_{1}}^{N} f(0)\right|
$$

$\forall \varepsilon>0$, by (3.1.7), there exists some $N\left(t_{1}, t_{2}\right) \in \mathbf{N}$ such that as $N>N\left(t_{1}, t_{2}\right)$

$$
\left|P_{t_{2}} f(0)-P_{t_{2}}^{N} f(0)\right|+\left|P_{t_{1}} f(0)-P_{t_{1}}^{N} f(0)\right|<\varepsilon .
$$

By (3.1.2), one has, with some large $A>0$ to be determined later,

$$
\begin{aligned}
\left|P_{t_{2}}^{N} f(0)-P_{t_{1}}^{N} f(0)\right| \leq & \left|S_{t_{2}} f(0)-S_{t_{1}} f(0)\right|+\mid \int_{0}^{t_{2}} S_{t_{2}-s} \sum_{i \in \Gamma_{N}} U_{i} \partial_{i} P_{s}^{N} f(0) d s \\
& \quad-\int_{0}^{t_{1}} S_{t_{1}-s} \sum_{i \in \Gamma_{N}} U_{i} \partial_{i} P_{s}^{N} f(0) d s \mid \\
\leq & \left|S_{t_{2}} f(0)-S_{t_{1}} f(0)\right| \\
& +\left|\int_{A}^{t_{2}} S_{t_{2}-s} \sum_{i \in \Gamma_{N}} U_{i} \partial_{i} P_{s}^{N} f(0) d s\right|+\left|\int_{A}^{t_{1}} S_{t_{1}-s} \sum_{i \in \Gamma_{N}} U_{i} \partial_{i} P_{s}^{N} f(0) d s\right| \\
& +\left|\int_{0}^{A} S_{t_{2}-s} \sum_{i \in \Gamma_{N}} U_{i} \partial_{i} P_{s}^{N} f(0) d s-\int_{0}^{A} S_{t_{1}-s} \sum_{i \in \Gamma_{N}} U_{i} \partial_{i} P_{s}^{N} f(0) d s\right|
\end{aligned}
$$


As for the term $\left|S_{t_{2}} f(0)-S_{t_{1}} f(0)\right|$, since $f \in \mathcal{D}^{\infty}, S_{t}$ is a finite product semigroup. Thus, by the same arguments as in proving (2.3.31), we have

$$
\left|S_{t_{2}} f(0)-S_{t_{1}} f(0)\right| \rightarrow 0 \quad\left(t_{1}, t_{2} \rightarrow \infty\right)
$$

According to (3.2.6) and $1-\eta-\gamma-\theta>0$, there exists some $A_{0}>0$ (independent of $N$ ) such that if $A \geq A_{0}$

$$
\begin{aligned}
& \left|\int_{A}^{t_{2}} S_{t_{2}-s} \sum_{i \in \Gamma_{N}} U_{i} \partial_{i} P_{s}^{N} f(0) d s\right|+\left|\int_{A}^{t_{1}} S_{t_{1}-s} \sum_{i \in \Gamma_{N}} U_{i} \partial_{i} P_{s}^{N} f(0) d s\right| \\
\leq & \sup _{i}\left\|U_{i}\right\|\left(\int_{A}^{t_{2}} \sum_{i \in \Gamma_{N}}\left\|\partial_{i} P_{s}^{N} f\right\| d s+\int_{A}^{t_{1}} \sum_{i \in \Gamma_{N}}\left\|\partial_{i} P_{s}^{N} f\right\| d s\right) \\
< & \varepsilon .
\end{aligned}
$$

As for the term in the last line of (3.2.17), firstly, since $\gamma=\sum_{i \in \mathbf{Z}^{d}}\left\|U_{i}\right\|<\infty$, there exists some $\Delta \subset \subset \mathbf{Z}^{d}$ such that

$$
\sum_{i \in \mathbf{Z}^{d} \backslash \Delta}\left\|U_{i}\right\| \leq \varepsilon
$$

secondly, by the same method to obtaining (2.3.31), there exists some $T>0$ such that as $t_{2}>t_{1}>T$

$$
\left|\int_{0}^{A} S_{t_{2}-s} \sum_{i \in \Delta} U_{i} \partial_{i} P_{s}^{N} f(0) d s-\int_{0}^{A} S_{t_{1}-s} \sum_{i \in \Delta} U_{i} \partial_{i} P_{s}^{N} f(0) d s\right|<\varepsilon
$$

hence

$$
\begin{aligned}
& \left|\int_{0}^{A} S_{t_{2}-s} \sum_{i \in \Gamma_{N}} U_{i} \partial_{i} P_{s}^{N} f(0) d s-\int_{0}^{A} S_{t_{1}-s} \sum_{i \in \Gamma_{N}} U_{i} \partial_{i} P_{s}^{N} f(0) d s\right| \\
& \leq\left|\int_{0}^{A} S_{t_{2}-s} \sum_{i \in \Delta} U_{i} \partial_{i} P_{s}^{N} f(0) d s-\int_{0}^{A} S_{t_{1}-s} \sum_{i \in \Delta} U_{i} \partial_{i} P_{s}^{N} f(0) d s\right| \\
& \quad+\left|\int_{0}^{A} S_{t_{2}-s} \sum_{i \in \Gamma_{N} \backslash \Delta} U_{i} \partial_{i} P_{s}^{N} f(0) d s\right|+\left|\int_{0}^{A} S_{t_{1}-s} \sum_{i \in \Gamma_{N} \backslash \Delta} U_{i} \partial_{i} P_{s}^{N} f(0) d s\right| \\
& \left.\leq \varepsilon+2 \varepsilon \int_{0}^{A}\left|\| P_{s}^{N} f\right||| d s \quad \text { (because } \sum_{i \in \mathbf{Z}^{d} \backslash \Delta}|| U_{i} \| \leq \varepsilon\right) \\
& \leq \varepsilon+2 \varepsilon \int_{0}^{A} e^{-(1-\eta-\gamma-\theta) s}|||f| \| d s \leq\left(1+\frac{2|\|f \mid\|}{1-\eta-\gamma-\theta}\right) \varepsilon .
\end{aligned}
$$

Combining (3.2.15)-(3.2.20), we conclude the proof.

Proof of Theorem IV: It is sufficient to prove that the limit is true for every $x$ in one ball $B_{R, \rho}$. By triangle inequality, it is obvious to have

$$
\begin{aligned}
\left|P_{t} f(x)-a\right| \leq & \left|P_{t} f(x)-P_{t}^{N} f(x)\right|+\left|P_{t}^{N} f(x)-P_{t}^{N} f(0)\right| \\
& +\left|P_{t}^{N} f(0)-P_{t} f(0)\right|+\left|P_{t} f(0)-a\right|
\end{aligned}
$$


$\forall \varepsilon>0$, by lemma 3.2.3, $\exists T_{0}>0$ such that, as $t>T_{0}$,

$$
\left|P_{t} f(0)-a\right|<\varepsilon \text {. }
$$

By Theorem III, $\forall t>0, \exists N(t) \in \mathbf{N}$ such that as $N>N(t)$

$$
\left|P_{t} f(x)-P_{t}^{N} f(x)\right|<\varepsilon,\left|P_{t}^{N} f(0)-P_{t} f(0)\right|<\varepsilon .
$$

Define an order for $\mathbf{Z}^{d}$ by its its lexicographic order, which is a one by one function $j: \mathbf{Z}^{d} \rightarrow \mathbf{Z}^{+}$, and denote the inverse function of $j$ by $i=i(j) \quad \forall j \in \mathbf{Z}^{+}$. For every $x \in \Omega$, we first arrange it according to the lexicographic order of $\mathbf{Z}^{d}$ and have $x=\left(x_{i(1)}, \ldots, x_{i(j)}, \ldots\right)$, then defines $x^{0}=0$ and $x^{j}=\left(x_{i(1)}, \ldots, x_{i(j)}, 0, \ldots, 0, \ldots,\right)$. (We often drop the $j$ in $i(j)$ if no confusion arises.) Recalling $B_{R, \rho}=\left\{x:\left|x_{i}\right| \leq R|i|^{\rho}\right\} \quad(R>$ $0, \rho>0)$, one has

$$
\begin{aligned}
& \left|P_{t}^{N} f(x)-P_{t}^{N} f(0)\right| \leq \sum_{j=1}^{\infty}\left|P_{t}^{N} f\left(x^{j}\right)-P_{t}^{N} f\left(x^{j-1}\right)\right| \leq \sum_{j=1}^{\infty}\left|x_{i(j)}\right| \cdot\left\|\partial_{i(j)} P_{t}^{N} f\right\| \\
\leq & \sum_{j=1}^{\infty} R \cdot|i(j)|^{\rho}\left\|\partial_{i(j)} P_{t}^{N} f\right\| \leq \sum_{j>l t^{d}} R \cdot j^{\rho}\left\|\partial_{i} P_{t}^{N} f\right\|+\sum_{j \leq l t^{d}} R \cdot j^{\rho}\left\|\partial_{i} P_{t}^{N} f\right\|,
\end{aligned}
$$

where $l \geq 1$ is some constant to be determined later and the last inequality is because of $|i(j)| \leq j$. On the one hand, as $t \rightarrow \infty$,

$$
\sum_{j \leq l t^{d}} R \cdot j^{\rho}\left\|\partial_{i} P_{t}^{N} f\right\| \leq R\left(l t^{d}\right)^{\rho+1} \mid\left\|P_{t}^{N} f\right\| \| \leq R\left(l t^{d}\right)^{\rho+1} e^{-t(1-\gamma-\theta-\eta)} \rightarrow 0 .
$$

On the other hand, by $(3.2 .2)$, as $l>(2 \operatorname{diam}(\Lambda) \cdot B \cdot t)^{d}\left(\right.$ thus $N_{i}=\frac{\operatorname{dist}(i, \Lambda(f))}{\operatorname{diam}(\Lambda)}>B t$ as $t$ is sufficiently large), we have

$$
\sum_{j>l t^{d}} R \cdot j^{\rho}\left\|\partial_{i} P_{t}^{N} f\right\| \leq R \sum_{j \geq l t^{d}} j^{\rho} e^{-A t-A N_{i}}\left\||f\|\|=K\|\| f \|| e^{-A t},\right.
$$

where $K=R \sum_{j \geq l t^{d}} j^{\rho} e^{-A N_{i}} \leq R \sum_{j \geq 0} j^{\rho} \exp \left\{-A \frac{j^{\frac{1}{d}}}{2 \cdot \operatorname{diam}(\Lambda)}\right\}<\infty$. Combining (3.2.24) and (3.2.25), we have some $T_{1}>0$ such that as $t>T_{1}$

$$
\left|P_{t}^{N} f(x)-P_{t}^{N} f(0)\right|<\varepsilon
$$

Take $T=\max \left\{T_{0}, T_{1}\right\}$, and combine (3.2.22), (3.2.23) and (3.2.26), we have that

$$
\left|P_{t} f(x)-a\right|<4 \varepsilon, \quad \text { as } t>T \text {. }
$$

\section{Appendix: Formal Derivation of (2.1.4)}

Suppose that Fourier transforms for the solution $u(t)$ and $f$ exist, then the equation for their Fourier transforms is

$$
\left\{\begin{array}{l}
\partial_{t} \hat{u}=-|\lambda|^{\alpha} \hat{u}+\hat{u}+\lambda \partial_{\lambda} \hat{u} \\
\hat{u}(0)=\hat{f}
\end{array}\right.
$$


where ' ${ }^{\prime}$ ' denotes the Fourier transform of functions. Suppose $\lambda>0$, set $\nu=\ln \lambda, \hat{v}=$ $e^{-t} \hat{u}\left(e^{\nu}\right), \quad \hat{g}(\nu)=\hat{f}\left(e^{\nu}\right)$, we have

$$
\left\{\begin{array}{l}
\partial_{t} \hat{v}=-e^{\alpha \nu} \hat{v}+\partial_{\nu} \hat{v} \\
\hat{v}(0)=\hat{g}(\nu)
\end{array}\right.
$$

Suppose $\hat{g}$ is positive, set $\hat{w}=\ln \hat{v}$, the equation for $\hat{w}$ is

$$
\left\{\begin{array}{l}
\partial_{t} \hat{w}=-e^{\alpha \nu}+\partial_{\nu} \hat{w} \\
\hat{v}(0)=\ln \hat{g}(\nu)
\end{array}\right.
$$

It is easy to solve the above equation by $\hat{w}(t)=\ln \hat{g}(\nu+t)-e^{\alpha \nu} \frac{e^{\alpha t}-1}{\alpha}$, thus

$$
\hat{w}(t)=\hat{g}(\nu+t) \exp \left\{-e^{\alpha \nu} \frac{e^{\alpha t}-1}{\alpha}\right\}
$$

and

$$
\hat{u}(t)=\hat{g}(\nu+t) \exp \left\{t-e^{\alpha \nu} \frac{e^{\alpha t}-1}{\alpha}\right\}=\hat{f}\left(e^{t} \lambda\right) \exp \left\{t-|\lambda|^{\alpha} \frac{e^{\alpha t}-1}{\alpha}\right\} .
$$

Hence, by Parseval's Theorem, we have

$$
\begin{aligned}
u(t) & =\frac{1}{\sqrt{2 \pi}} \int_{\mathbf{R}} \hat{f}\left(e^{t} \lambda\right) \exp \left\{t-|\lambda|^{\alpha} \frac{e^{\alpha t}-1}{\alpha}\right\} e^{i \lambda x} d \lambda \\
& =\int_{\mathbf{R}} \hat{f}(\lambda) \frac{1}{\sqrt{2 \pi}} \exp \left\{-|\lambda|^{\alpha} \frac{1-e^{-\alpha t}}{\alpha}+i \lambda e^{-t} x\right\} d \lambda=\int_{\mathbf{R}} p\left(\frac{1-e^{-\alpha t}}{\alpha} ; e^{-t} x, y\right) f(y) d y
\end{aligned}
$$

\section{References}

[1] S. Albeverio, B. Rüdiger, and J.-L. Wu Invariant Measures and Symmerty Property of Levy Type Operator, Potential Analysis 13: 147-168, 2000.

[2] D. Bakry and M. Emery, Diffusions hypercontractives, Séminaire de Probabilités XIX, Lecture Notes in Math. 1123, p.177-206, Springer, 1985.

[3] K. Bichteler, Stochastic Integration with Jumps, Encyclopedia of Mathematics and its Applications, No. 89, Cambridge, 2002.

[4] V. I. Bogachev, Measure Theory, Volume II, Springer, Berlin, 2007.

[5] Z.-Q. Chen and T. Kumagai, Heat kernel estimates for stable-like processes on d-sets, Stoch. Proc. Their Appl., 108 (2003), no. 1, 27-62.

[6] G. Da Prato and J. Zabczyk, Ergodicity for Infinite Dimensional Systems (LMS Lecture Note series 229), Cambridge University Press.

[7] A. Guionnet and B. Zegarlinski, Lectures on Logarithmic Sobolev Inequality, Séminaire de Probabilitiés XXXVI, Lecture Notes in Math. 1801, 1-134, Springer, Berlin, (2003). 
[8] N. Jacob, Pseudo-Differential Operators and Markov Processes. Vol. 1: Fourier Analysis and Semigroups. Imperial College Press, London, 2001.

[9] N. Jacob, Pseudo-Differential Operators and Markov Processes. Vol. 3: Markov Processes and Applications. Imperial College Press, London, 2005.

[10] T. M. Liggett, Interacting Particle Systems, Grundlehren der mathematischen Wissenschaften, 276 (1985) Berlin-Heidelberg-New York: Springer

[11] S. Peszat, J. Zabczyk, Stochastic Parital Differential Equations With Levy Noise Cambridge University Press, 2007, XII + 419 pp. .

[12] K. Yosida, Functional Analysis, 6th edition, Springer-Verlag, Berlin.

[13] B. Zegarlinski, Strong Decay to Equilibrium for the Stochastic Dynamics of Unbounded Spin Systems on a Lattice, Commun. Math. Phys. 175, 401-432 (1996).

Lihu Xu: EURANDOM, P.O. Box 513 - 5600 MB Eindhoven, The Netherlands, xu@eurandom.tue.nl.

Boguslaw Zegarlinski: Mathematics Department, Imperial College London, SW7 2AZ, United Kingdom, b.zegarlinski@imperial.ac.uk. 